\title{
ON GRITICAL GROUPS
}

\author{
L. G. KOVÁCS and M. F. NEWMAN
}

(Received 6 September 1965)

\section{Introduction}

The concept of critical group was introduced by D. C. Cross (as reported by G. Higman in [5]): a finite group is called critical if it is not contained in the variety generated by its proper factors. (The factors of a group $G$ are the groups $H / K$ where $K \leqq H \leqq G$, and $H / K$ is a proper factor of $G$ unless $H=G$ and $K=1$ ). Some investigations concerning finite groups and varieties depend on the investigator's ability to decide whether a given group is critical or not. (For instance, one of the crucial points in the important paper [9] of Sheila Oates and M. B. Powell is a necessary condition of criticality: their Lemma 2.4.2.) An obvious necessary condition is that the group should have only one minimal normal subgroup: the group is then called monolithic, and the minimal normal subgroup its monolith. This is, however, far from being a sufficient condition, and it is the purpose of the present paper to give some sufficient conditions for the criticality of monolithic groups. (We consider the trivial group neither monolithic nor critical.) The basis of our results is an analysis of the following situation.

(1.1) Let $\mathfrak{D}$ be a finite set of finite groups which is factor-closed in the sense that every factor of every group in $\mathscr{D}$ is isomorphic to some group in $\mathfrak{D}$, and let $G$ be a finite monolithic group in the variety var $\mathscr{D}$ generated by $\mathscr{D}$. Let $\subseteq$ be the set of those finite sequences $D_{1}, \cdots, D_{n}$ of non-trivial elements of $D$ for which the direct product $\Pi\left(D_{i}: 1 \leqq i \leqq n\right)$ has a factor isomorphic to $G$. By Lemma 4.3 of Higman [4], $S$ is not empty. Associate with each sequence in $\mathbb{S}$ the sequence of the orders of its terms rearranged if necessary so that these numbers occur in non-increasing order. This defines a map from $\subseteq$ to the lexicographically well-ordered set of all finite non-increasing sequences of positive integers. Let $D_{1}, \cdots, D_{n}$ be a counterimage of the first element of the image of $\mathfrak{S}$. Then each $D_{i}$ is critical:

if, say, $D_{n}$ were not critical, by the lemma of Higman quoted above $D_{n}$ would be isomorphic to a factor of the direct product of some finite sequence 
$D_{n 1}, \cdots, D_{n k}$ of elements of $\mathfrak{D}$ isomorphic to proper non-trivial factors of $D_{n}$, and then $D_{1}, \cdots, D_{n-1}, D_{n 1}, \cdots, D_{n k}$ would be an element of $\subseteq$ whose image preceded that of $D_{1}, \cdots, D_{n}$.

Our purpose is now to derive connections between $G$ and the critical groups $D_{1}, \cdots, D_{n}$.

Some terminology is required for stating these. For any finite group $X$, let $\sigma X$ denote the socle (that is, the product of the minimal normal subgroups) of $X$ (thus $\sigma X=1$ if and only if $X=1$ ), and let $\sigma^{*} X$ be the centralizer of $\sigma X$ in $X$. By an abstract subgroup function we shall mean a function $\mu$ defined on the class of all finite groups and such that $\mu X$ is a subgroup of $X$ and $(\mu X) \alpha=\mu(X \alpha)$ for every finite group $X$ and every isomorphism $\alpha: X \longmapsto X \alpha$. (Examples: $\left.\sigma, \sigma^{*}\right)$. If $Y$ is a normal subgroup of a finite group $X$, then $\mu(X \div Y)$ is the subgroup of $X$ defined by $\mu(X \div Y) / Y=\mu(X / Y)$. A subdirect function is an abstract subgroup function $\mu$ with the property that $\mu(X \div Y \cap Z)=\mu(X \div Y) \cap \mu(X \div Z)$ whenever $Y, Z$ are normal subgroups of a finite group $X$.

First, we shall prove that in the above set-up

$$
\text { each } \sigma D_{i} \text { is similar to } \sigma G
$$

in the sense of [6]: that is, there exist isomorphisms $\theta_{i}: \sigma G \longmapsto \sigma D_{i}$ and $\varphi_{i}: G / \sigma^{*} G \longmapsto D_{i} / \sigma^{*} D_{i}$ such that $\left(x^{\nu}\right) \theta_{i}=\left(x \theta_{i}\right)^{z}$ whenever $x \in \sigma G, y \in G$, and $z \in\left(y \sigma^{*} G\right) \psi_{i}$.

Next, two comparatively simple observations:

$$
G / \sigma G \in \operatorname{var}\left\{D_{i} / \sigma D_{i}: 1 \leqq i \leqq n\right\},
$$

and

$$
\sigma^{*} G \in \operatorname{var}\left\{\sigma^{*} D_{i}: 1 \leqq i \leqq n\right\}
$$

Finally,

(1.15) if $\mu$ is a subdirect function and $\sigma^{*} D_{i} \leqq \mu D_{i}$ for $i=1, \cdots, n$, then also $\sigma^{*} G \leqq \mu G$.

We give three direct applications of these results. First, we give new proofs of Theorems 1 and 2 of [6]:

(1.2) If a finite monolithic group is not critical, then it is contained in the variety generated by its proper subgroups.

(1.3) A finite group is contained in the variety generated by its proper factor groups if and only if it is not monolithic.

The third result confirms a conjecture of Sheila Oates:

(1.4) THEOREM. If $G$ is a finite monolithic group and $G$ is not critical, 
then $G / \sigma G$ is contained in the variety generated by the proper subgroups of $G / \sigma G$.

We are grateful to Dr Oates for telling us of this conjecture and of the steps she had taken towards confirming it.

Note that 1.2 and 1.4 can be thought of as sufficient conditions for the criticality of monolithic groups. The main results of the paper are expressed in the next two theorems. The first is a general one and is an immediate consequence of the discussion so far; the second is more specific, and is derived from the first without further reference to the above analysis. (The reader will note that in proving the second the full power of the first is never used.)

(1.5) Theorem. Let $G$ be a finite monolithic group, D the set of all factor groups of proper subgroups of $G$, and $\mathfrak{D}^{*}$ the subset of those critical groups in $\mathfrak{D}$ whose monoliths are similar to the monolith $\sigma G$ of $G$. If $G$ is not critical, then

(1.51) $G \in \operatorname{var} \mathfrak{D}^{*}$,

(1.52) $G / \sigma G \in \operatorname{var}\left\{D / \sigma D: D \in D^{*}\right\}$,

(1.53) $\sigma^{*} G \in \operatorname{var}\left\{\sigma^{*} D: D \in \mathfrak{D}^{*}\right\}$, and

(1.54) $\sigma^{*} G \leqq \mu G$ whenever $\mu$ is a subdirect function such that $\sigma^{*} D \leqq \mu D$ for every $D$ in $D^{*}$.

The first of these statements follows from $1.2,1.11,1.12$, and the rest from 1.13, 1.14, 1.15.

(1.6) THeOREm. Let $G$ be a finite monolithic group. If $G$ satisfies any one of the following conditions, then $G$ is critical:

(1.61) $\sigma^{*} G \leqq \sigma G$;

(1.62) the Frattini subgroup $\Phi(G)$ of $G$ is trivial;

(1.63) $\Phi(G)=\sigma^{*} G$;

(1.64) $\sigma^{*} G$ is nilpotent and has only one maximal G-normal subgroup;

(1.65) $\sigma^{*} G$ is abelian and has no elements of prime order outside $\sigma G$;

(1.66) $G$ has abelian Sylow $p$-subgroups for some prime $p$ which divides the order of $\sigma G$.

The first three parts will be proved from 1.51. (The special case $\sigma^{*} G=1$ of 1.61 seems to be the content of Theorem 1 of $P$. M. Weichsel [11].) In proving 1.64, we shall use also 1.54 , the relevant subdirect function being an iterate $\sigma_{n}$ of $\sigma$ (defined inductively by $\sigma_{1}=\sigma, \sigma_{i}(X)=\sigma\left(X \div \sigma_{i-1} X\right)$ if $i>1)$. The fact that $\sigma$ and its iterates are subdirect will be proved in Section 2 , along with some other information on subdirect functions intended to facilitate possible further applications of 1.54 . It will be shown that 1.65 
follows from 1.64. We are indebted to Mr P. J. Cossey for pointing out that 1.66 follows easily from 1.65 if the solubility of $G$ is also assumed, and to Dr $Z$. Janko for a suggestion which enabled us to eliminate from the argument the assumption of solubility.

Originally we proved 1.65 by a different method; as this gives further insight, we briefly indicate how the old proof ran. It was based on the use of the laws $u_{n}=1$ where

$$
\begin{array}{ll}
u_{3}=\left[\left(x_{1}^{-1} x_{2}\right)^{x_{1,3}},\left(x_{1}^{-1} x_{3}\right)^{x_{1,3}},\left(x_{2}^{-1} x_{3}\right)^{x_{1,3}}\right], & \text { and } \\
u_{n}=\left[u_{n-1},\left(x_{1}^{-1} x_{n}\right)^{x_{1, n}}, \cdots,\left(x_{n-1}^{-1} x_{n}\right)^{x_{n-1, n}}\right] & \text { if } n>\mathbf{3} .
\end{array}
$$

These laws have, in the meanwhile, proved very useful in obtaining a simplified proof of the main result of Oates and Powell [9]; in particular, we gave in [7] the following lemmas ( $n \geqq 3$ being assumed throughout):

(1.71) (4.3 of [7]) If $H$ is any group of order less than $n$, then $u_{n}=1$ is a law in $H$.

(1.72) (4.4 of [7]) If the centralizer of a chief factor of $H$ is of index at least $n$ in $H$, then $u_{n}=1$ is not a law in $H$.

We had one more:

(1.73) If $H$ has an abelian normal subgroup of index less than $n-1$, then $u_{n}=1$ is a law in $H$.

It was easy to prove from these lemmas that,

(1.74) under the assumptions of 1.65 , if $n$ is the order of $G / \sigma^{*} G$ and $e$ is the exponent of $\sigma^{*} G / \sigma G$, then $\left(u_{n}\right)^{6}=1$ is a law in every proper factor of $G$ but not in $G$ itself,

so that $G$ is critical. (For the trivial cases of $1.74, u_{1}=x, u_{2}=[x, y]$.)

It is interesting to note that, when $\sigma^{*} G$ is abelian, 1.64 is often a necessary as well as a sufficient condition of criticality:

(1.8) Theorem. Let $G$ be a critical group, with $\sigma^{*} G$ abelian and $\sigma G$ a p-group. Unless $\sigma^{*} G$ is precisely the largest p-soluble normal subgroup of $G$ and $\sigma^{*} G<G$, it followes that $\sigma^{*} G$ has only one maximal $G$-normal subgroup.

This will be proved by first showing (with the help of a result from Higman [3]) that $\sigma^{*} G$ is complemented in $G$, and then applying a necessary condition of criticality (Theorem 9 in Higman [5]). We expect that the qualifications cannot be omitted from this theorem; that is, the conclusion will fail to hold in some groups where $\sigma^{*} G$ is the largest $p$-soluble normal subgroup of $G$ and $\sigma^{*} G<G$. It is easy to see that the theorem fails, even for soluble $G$, if $\sigma^{*} G$ is only assumed nilpotent of class 2. Indeed, it is likely that 
a necessary and sufficient condition for the criticality of monolithic groups $G$ with abelian or nilpotent $\sigma^{*} G$ would have to be sought in an entirely different setting.

Finally, we remark that critical $p$-groups have been studied by Weichsel in [12].

\section{Subdirect functions}

Recall from the introduction that, in this paper, an abstract subgroup function is a function $\mu$ defined on the class of all finite groups and such that $\mu X \leqq X$ and $\mu(X \alpha)=(\mu X) \alpha$ for every finite group $X$ and every isomorphism $\alpha: X \gg X \alpha$. (In particular, $\mu X$ is always a characteristic subgroup of $X$ ). Also, if $Y \leqq X$, then the (normal) subgroup $\mu(X \div Y)$ of $X$ is defined by $\mu(X \div Y) / Y=\mu(X / Y)$. (Note that $Y \leqq \mu(X \div Y)$.) We shall need the following technical remark:

$$
\text { If } U, V \leqq X, U \leqq V, \text { then } \mu(X \div V) / U=\mu(X / U \div V / U) .
$$

Proof. If $\alpha: \frac{X / U}{V / U} \succ \frac{X}{V}$ is the natural isomorphism, then

$$
\begin{aligned}
\frac{\mu(X \div V) / U}{V / U} & =\frac{\mu(X \div V) / U}{V / U} \alpha \alpha^{-1}=\frac{\mu(X \div V)}{V} \alpha^{-1} \\
& =\mu(X / V) \alpha^{-1}=\mu \frac{X / U}{V / U}=\frac{\mu(X / U \div V / U)}{V / U} .
\end{aligned}
$$

For two abstract subgroup functions $\mu, \nu$ and a finite group $X$, define $\mu^{\nu} X=\nu(X \div \mu X)$. It is easy to check that $\mu^{\nu}$ (in the terminology of $\mathrm{R}$. Baer [1], the "extension" of $\mu$ by $\nu$ ) is an abstract subgroup function: if $\alpha: X \gg X \alpha$ is an isomorphism and $\beta: X / \mu X \multimap X \alpha / \mu X \alpha$ is the isomorphism induced by $\alpha$, then

$$
\begin{aligned}
\frac{\mu^{\nu}(X \alpha)}{\mu X \alpha} & =\frac{\nu(X \alpha \div \mu X \alpha)}{\mu X \alpha}=\nu \frac{X \alpha}{\mu X \alpha}=\nu \frac{X}{\mu X} \beta=\frac{\nu(X \div \mu X)}{\mu X} \beta \\
& =\frac{\mu^{\nu} X}{\mu X} \beta=\frac{\left(\mu^{\nu} X\right) \alpha}{\mu X \alpha} .
\end{aligned}
$$

We shall need a further technical remark:

$$
\mu^{\nu}(X \div Y)=\nu(X \div \mu(X \div Y))
$$

Proof. Apply 2.1 with $v$ in place of $\mu$ and $U=Y, V=\mu(X \div Y)$ :

$$
\begin{aligned}
\nu(X \div \mu(X \div Y)) / Y & =\nu(X / Y \div \mu(X \div Y) / Y)=\nu(X / Y \div \mu(X / Y)) \\
& =\mu^{\nu}(X / Y)=\mu^{\nu}(X \div Y) / Y .
\end{aligned}
$$


It follows now that

(2.3) the operation of extension is associative.

Proof. Apply 2.2 with $Y=\lambda X$ :

$\lambda^{\left(\mu^{\nu}\right)} X=\mu^{\nu}(X \div \lambda X)=\nu(X \div \mu(X \div \lambda X))=\nu\left(X \div \lambda^{\mu} X\right)=\left(\lambda^{\mu}\right)^{\nu} X$.

This has also been proved by Baer [1] (Lemma 1.8): this section so far has much in common with his paper. We need one further term used by Baer: if $\mu$ is an abstract subgroup function, its $i$-th iterate $\mu_{i}$ is defined (for each non-negative integer $i$ ) inductively by $\mu_{0}=o$ (the trivial subgroup function, $o X=1$ for every finite group $X)$ and $\mu_{i+1}=\left(\mu_{i}\right)^{\mu}$. Note that $\mu_{1}=\mu$.

Recall from the introduction that an abstract subgroup function $\mu$ is called a subdirect function if $\mu(X \div Y \cap Z)=\mu(X \div Y) \cap \mu(X \div Z)$ whenever $Y, Z \leqq X$ and $X$ is a finite group. A routine induction shows that

(2.4) if $\mu$ is a subdirect function and $Y_{1}, \cdots, Y_{n} \leqq X$, then $\mu\left(X \div Y_{1} \cap \cdots \cap Y_{n}\right)=\mu\left(X \div Y_{1}\right) \cap \cdots \cap \mu\left(X \div Y_{n}\right)$.

(2.5) If $\mu$ is a subdirect function and $Y \leqq X$, then $\mu X \leqq \mu(X \div Y)$.

This follows from the definition of subdirect function if one puts $Z=1$.

(2.6) If $\mu, v$ are subdirect functions, so is $\mu^{\nu}$.

Proof. Let $Y, Z \leqq X$. Then, from 2.2,

$$
\begin{aligned}
\mu^{\nu}(X \div Y \cap Z) & =v(X \div \mu(X \div Y \cap Z)) \\
& =v(X \div(\mu(X \div Y) \cap \mu(X \div Z))) \\
& =v(X \div \mu(X \div Y)) \cap \nu(X \div \mu(X \div Z)) \\
& =\mu^{\nu}(X \div Y) \cap \mu^{\nu}(X \div Z) .
\end{aligned}
$$

Consequently, as $o$ is obviously subdirect,

(2.7) if $\mu$ is a subdirect function, so are all its iterates.

(2.8) The socle function $\sigma$ is subdirect.

Proof. Suppose that $X$ and its normal subgroups $Y, Z$ provide a counterexample:

$$
\sigma(X \div Y \cap Z) \neq \sigma(X \div Y) \cap \sigma(X \div Z) .
$$

Using 2.1, this inequality can be re-written as

$$
\sigma(X / Y \cap Z) \neq \sigma(X / Y \cap Z \div Y / Y \cap Z) \cap \sigma(X / Y \cap Z \div Z / Y \cap Z) .
$$

Hence there is a counterexample (namely $X / Y \cap Z, Y / Y \cap Z, Z / Y \cap Z$ ) in which the two normal subgroups are disjoint. With a shift of notation if 
necessary take $X, Y, Z$ to be a counterexample of this kind; that is, assume that $Y \cap Z=1$ and $\sigma X \neq \sigma(X \div Y) \cap \sigma(X \div Z)$. Call this intersection $D$.

Since the natural homomorphism $X \rightarrow X / Y$ maps minimal normal subgroups of $X$ onto trivial or minimal normal subgroups of $X / Y, Y \sigma X / Y$ $\leqq \sigma(X / Y)$, so that $\sigma X \leqq \sigma(X \div Y)$. The same holds for $Z$ in place of $Y$; therefore we must have $\sigma X<D$. Let $N$ be minimal among the normal subgroups of $X$ which are contained in $D$ but not in $\sigma X$, and let $M$ be a minimal normal subgroup of $X$ in $N$. As $Y \cap Z=1, M \$ Y \cap Z$ : say, $M \$ Y$. Then $M \cap Y=1$, and $M Y / Y$ is a minimal normal subgroup of $X / Y$. The intersection of the maximal $X / Y$-normal subgroups of $\sigma(X / Y)$ is trivial; hence for one of them, say, for $K / Y, M Y / Y \cap K / Y=1$ and $(M Y / Y)(K / Y)$ $=\sigma(X / Y)$. This, with $M \cap Y=1$, implies that $M \cap K=1$ and $M K=$ $\sigma(X \div Y)$. Since $M<N \leqq D \leqq \sigma(X \div Y)=M K, N=M(N \cap K)$ and, of course, $M \cap(N \cap K)=1$. In particular, $N \cap K<N$; as $N$ was chosen minimal, $N \cap K \leqq \sigma X$; on the other hand, $M \leqq \sigma X$, so $N=M(N \cap K)$ $\leqq \sigma X$. This contradicts the assumption $N \leqq \sigma X$ made when $N$ was chosen, and the proof is complete.

In view of 2.7, we now have the particular result needed in the sequel:

(2.9) The iterates $\sigma_{i}$ of the socle function $\sigma$ are subdirect functions.

\section{Proofs of $1.12-1.15$}

Recall the relevant part of the set-up of 1.1: We have a finite monolithic group $G$ and a direct product $P$ of a suitably chosen finite sequence $D_{1}, \cdots, D_{n}$ of non-trivial finite groups such that some factor, say $S / T$, of $P$ is isomorphic to $G$. To exclude a trivial case, assume that $n>1$. For simplicity of expression, identify $G$ with $S / T$. The choice of $P$ implies that if any term of the sequence $D_{1}, \cdots, D_{n}$ is omitted or replaced by a proper factor of itself, the direct product of the resulting sequence no longer has a factor isomorphic to $G$. We shall refer to this by saying that $P$ is minimal. $L$ et $K_{i}$ be the kernel of the canonical projection of $P$ onto $D_{i}$, that is, $K_{i}=\prod\left(D_{j}: 1 \leqq j \leqq n, j \neq i\right)$. Throughout this section, statements involving $i$ are to be taken as made for all values of $i$ between 1 and $n$ (inclusive).

The analysis of this situation begins with some obvious steps.

(3.1) $S K_{i}=P$.

(3.2) A subgroup of $D_{i}$ is normal (in $D_{i}$ ) if and only if it is normalized by $S$.

(3.3) If $1<H \leqq D_{i}$, then $H \cap S>1$.

(3.4) $T \cap D_{i}=1$.

(3.5) If $N$ is a minimal no:mal subgroup of $D_{i}$, then $T N=\sigma(S \div T)$. 
Of these, 3.2 is an immediate consequence of 3.1 , while $3.1,3.3,3.4$ follow from the minimality of $P$, with 3.2 giving $T \cap D_{i} \leqq D_{i}$ for the proof of 3.4. As for the proof of 3.5, note that $N$ is a minimal normal subgroup of $S$ (in view of 3.2 and 3.3) and $T \cap N=1$ (because of 3.4); also, that $\sigma(S / T)$ is the only minimal normal subgroup of $S / T$, being the monolith of $G$. The next step is an immediate consequence of $\mathbf{3 . 5}$ :

(3.6) $\sigma(S \div T) \cap D_{i}=\sigma D_{i}$ with $\sigma D_{i}$ minimal normal in $D_{i}$, so that $D_{i}$ is monolithic; also $\sigma(S \div T)=T \sigma D_{i}$.

At this stage, 1.13 can already be derived. Namely, we have that $G / \sigma G=S / \sigma(S \div T)=S / T \sigma D_{i}=S / \Pi\left(T \sigma D_{i}: 1 \leqq i \leqq n\right)$, so that $G / \sigma G$ is isomorphic to a factor group of $S / \Pi\left(\sigma D_{i}: 1 \leqq i \leqq n\right)$, and the latter can naturally be considered a subgroup of $\Pi\left(D_{i} / \sigma D_{i}: 1 \leqq i \leqq n\right)$.

On the basis of 3.1-3.6, 2.1 and 2.2 of [6] could be invoked to prove 1.12; we prefer to make the present argument self-contained. For the proof of 1.12 , define the required mappings $\theta_{i}, \psi_{i}$ as follows. If $s T \in \sigma(S / T)=$ $\sigma G, 3.6$ and 3.4 enable us to write $s=t_{i} d_{i}$ with uniquely determined $d_{i}$ from $\sigma D_{i}$ and $t_{i}$ from $T$; let $\theta_{i}: s T \rightarrow d_{i}$. It is obvious that $\theta_{i}$ is an isomorphism of $\sigma G$ onto $\sigma D_{i}$. If $\left(s^{*} T\right) \sigma^{*}(S / T) \in(S / T) / \sigma^{*}(S / T)=G / \sigma^{*} G$, take $s^{*}=k_{i} d_{i}^{*}$ with $d_{i}^{*} \in D_{i}, k_{i} \in K_{i}$, according to the direct decomposition $K_{i} \times D_{i}$ of $P$, and put $\psi_{i}:\left(s^{*} T\right) \sigma^{*}(S / T) \rightarrow d_{i}^{*} \sigma^{*} D_{i}$. Using 3.6 and 3.7 below, it is straightforward to check that the $\psi_{i}$ are isomorphisms of $G / \sigma^{*} G$ onto the $D_{i} / \sigma^{*} D_{i}$ and that they fit together with the $\theta_{i}$ in the manner required for 1.12.

The next step will have 1.14 as a trivial corollary.

$$
\sigma^{*}(S \div T)=S \cap \prod\left(\sigma^{*} D_{i}: 1 \leqq i \leqq n\right)=S \cap K_{i} \sigma^{*} D_{i}
$$

Proof. Observe that

$$
\begin{aligned}
{\left[\sigma D_{i}, K_{i} \sigma^{*}(S \div T)\right] } & =\left[\sigma D_{i}, \sigma^{*}(S \div T)\right], \text { for even }\left[D_{i}, K_{i}\right]=1 \\
& \leqq\left[T \sigma D_{i}, \sigma^{*}(S \div T)\right] \cap \sigma D_{i}, \text { for } \sigma D_{i} \leqq P \\
& =\left[\sigma(S \div T), \sigma^{*}(S \div T)\right] \cap \sigma D_{i}, \text { by } 3.6 ; \\
& \leqq T \cap \sigma D_{i}, \text { by the definition of } \sigma^{*}(S \div T) \\
& =1, \text { by } 3.4
\end{aligned}
$$

Thus $K_{i} \sigma^{*}(S \div T) \cap D_{i} \leqq \sigma^{*} D_{i}$. If $s=\prod\left(d_{i}: 1 \leqq i \leqq n\right), d_{i} \in D_{i}$, according to the direct decomposition $\Pi\left(D_{i}: 1 \leqq i \leqq n\right)$ of $P$ and $s \in \sigma^{*}(S \div T)$, it follows that $d_{i} \in \sigma^{*} D_{i}$; in other words, we have proved that $\sigma^{*}(S \div T)$ $\leqq \prod\left(\sigma^{*} D_{i}: 1 \leqq i \leqq n\right)$. For obvious reasons, $\Pi\left(\sigma^{*} D_{i}: 1 \leqq i \leqq n\right) \leqq K_{i} \sigma^{*} D_{i}$. Finally, 3.6 and $\left[\sigma D_{i}, K_{i} \sigma^{*} D_{i}\right]=1$ imply that $\left[\sigma(S \div T), S \cap K_{i} \sigma^{*} D_{i}\right]$ $=\left[T \sigma D_{i}, S \cap K_{i} \sigma^{*} D_{i}\right] \leqq[T, S] \leqq T$, so that $S \cap K_{i} \sigma^{*} D_{i} \leqq \sigma^{*}(S \div T)$.

It remains to prove 1.15 . Let $\mu$ be a subdirect function such that $\sigma^{*} D_{i}$ $\leqq \mu D_{i}$ (for every value of $i$ ), and put $S \cap K_{i}=L_{i}$. Since the restriction to 
$S$ of the canonical projection $P \rightarrow D_{i}$ is an epimorphism $S \rightarrow D_{i}$ (cf. 3.1) with kernel $L_{i}$, we have that $\mu\left(S \div L_{i}\right)$ is the full counterimage of $\mu D_{i}$ in $S$ under this projection: that is, $\mu\left(S \div L_{i}\right)=S \cap K_{i} \mu D_{i}$. In view of 3.7 and $\sigma^{*} D_{i} \leqq \mu D_{i}$, it follows that $\sigma^{*}(S \div T) \leqq \mu\left(S \div L_{i}\right)$. As $\bigcap\left(L_{i}: 1 \leqq i \leqq n\right)=1,2.4$ gives that $\sigma^{*}(S \div T) \leqq \bigcap\left(\mu\left(S \div L_{i}\right): 1 \leqq i \leqq n\right)$ $=\mu S$, and then 2.5 ensures that $\sigma^{*}(S \div T) \leqq \mu(S \div T)$. Thus we have that $\sigma^{*} G \leqq \mu G$, as required.

\section{Proofs of $1.2-1.4$}

Proof of 1.2: reductio ad absurdum. Suppose that there exists a finite monolithic group $G$ which is not critical and is not contained in the variety generated by proper subgroups. Let $\mathfrak{D}_{1}$ be the set of proper factors of $G$, so that $\mathfrak{D}_{1}=\{H \mid K: K \unlhd H \leqq G, H / K \neq G / 1\}$, and let $\mathscr{D}_{2}$ be the set of factor groups of proper subgroups of $G$, that is, $\mathfrak{D}_{2}=\{H / K: K \unlhd H<G\}$. By our assumptions, $G \in \operatorname{var} \mathscr{D}_{1}$ but $G \notin \operatorname{var} \mathfrak{D}_{2}$. Obviously, $\mathfrak{D}_{2} \subset \mathscr{D}_{1}$. Let $\mathfrak{D}$ be a subset of $\mathfrak{D}_{1}$ which is minimal (in the inclusion-order of subsets) with respect to the following conditons:

(4.1) $\mathfrak{D}$ is factor-closed,

(4.2) $G \in \operatorname{var} \mathfrak{D}$,

(4.3) $\mathfrak{D}_{2} \subseteq \mathfrak{D}$.

(The set of those subsets of $\mathscr{D}_{1}$ which satisfy these conditions contains $\mathscr{D}_{1}$ and so is not empty). Then the difference set $\mathscr{D}-\mathscr{D}_{2}$ is not empty (for $\left.G \in \operatorname{var} \mathfrak{D}-\operatorname{var} \mathfrak{D}_{2}\right)$; let $D$ be a group of maximal order in $\mathscr{D}-\mathscr{D}_{2}$, and put $\mathscr{D}_{0}=\mathfrak{D}-\{D\}$. Note that every proper factor of every group in $\mathfrak{D}$ is isomorphic to some group in $\mathscr{D}_{0}$; also, that $\mathfrak{D}_{0}$ satisfies (4.1) and (4.3), and so must fail (4.2): $G \notin \operatorname{var} \mathscr{D}_{0}$. It follows from 1.13 that $G / \sigma G \in \operatorname{var} \mathscr{D}_{0}$. Hence every proper factor group of $G$, that is, every group in $\mathscr{D}_{1}-\mathfrak{D}_{2}$, lies in var $\mathscr{D}_{0}$ : thus $\mathscr{D}_{2} \subseteq \mathscr{D}_{0} \subseteq \mathscr{D}_{1}$ implies that var $\mathscr{D}_{0}=\operatorname{var} \mathscr{D}_{1}$. We conclude that $G \notin \operatorname{var} \mathscr{D}_{1}$, contrary to one of the initial assumptions. This completes the proof.

Proof of 1.3. The non-trivial part of the statement is that if $G$ is a finite monolithic group then $G$ is not contained in the variety generated by its proper factor groups. If $G$ is critical, there is nothing to prove; thus we assume that $G \in \operatorname{var} \mathscr{D}_{1}$ where $\mathfrak{D}_{1}=\{H / K: K \unlhd H \leqq G, H / K \neq G / 1\}$. Let $\mathfrak{D}$ be a subset of $D_{1}$ which is minimal with respect to 4.1 and 4.2 , let $D$ be a group of maximal order in $\mathfrak{D}$, and put $\mathfrak{D}_{0}=\mathfrak{D}-\{D\}$. Then every proper factor of every group in $\mathscr{D}$ is isomorphic to some group in $\mathscr{D}_{0}$; also, $\mathscr{D}_{0}$ is factor-closed and so we must have $G \notin \operatorname{var} \mathfrak{D}_{0}$. Now 1.13 gives that $G / \sigma G \in \operatorname{var} \mathfrak{D}_{0}$; we conclude that the variety generated by the proper factor groups of $G$ is contained in var $\mathscr{D}_{0}$ and hence cannot contain $G$. 
Proof of 1.4. Let $G$ be a finite, monolithic, non-critical group. By 1.2, $G \in \operatorname{var} \mathfrak{D}$ with $\mathscr{D}=\{H \mid K: K \leqq H<G\}$. Apply 1.1 to this $\mathscr{D}$ and $G$ : by 1.12 , there exist monolithic groups $D_{1}, \cdots, D_{n}$ in $D$ such that

$$
\left|\sigma D_{1}\right|=\cdots=\left|\sigma D_{n}\right|=|\sigma G| \text {. }
$$

As 1.13 also holds, 1.4 will be proved if we show that each $D_{i} / \sigma D_{i}$ is isomorphic to some factor of some proper subgroup of $G / \sigma G$. Let $D_{i}=H_{i} / K_{i}$ with $K_{i} \unlhd H_{i}<G$. First we show that $H_{i} \sigma G / \sigma G<G / \sigma G$, and then that $D_{i} / \sigma D_{i}$ is isomorphic to a factor of $H_{i} \sigma G / \sigma G$. For the first step, suppose that $H_{i} \sigma G=G$. Then $M \sigma G=G$ for any maximal subgroup $M$ of $G$ which contains $H_{i}$; thus $\sigma G \$ M$, and so $\sigma G \$ \Phi(G)$ : as $\sigma G$ is contained in every nontrivial normal subgroup of $G$, this implies that $\Phi(G)=1$. But 1.62 asserts that in this case $G$ is critical: a contradiction. [Naturally, this application of 1.62 will only be justified after we have proved 1.62 without using 1.4.] Hence indeed we must have $H_{i} \sigma G / \sigma G<G / \sigma G$. For the second step, note that as $\left(H_{i} \cap \sigma G\right) K_{i} / K_{i} \unlhd H_{i} / K_{i}$ and, by $4.4,\left|\left(H_{i} \cap \sigma G\right) K_{i}\right| K_{i}|\leqq| \sigma G|=| \sigma\left(H_{i} / K_{i}\right) \mid$ the fact that $\sigma\left(H_{i} / K_{i}\right)$ is contained in every non-trivial normal subgroup of $H_{i} / K_{i}$ implies that $\left(H_{i} \cap \sigma G\right) K_{i} / K_{i} \leqq \sigma\left(H_{i} / K_{i}\right)$. Thus $\left(H_{i} / K_{i}\right) / \sigma\left(H_{i} / K_{i}\right)$ is isomorphic to a factor of $H_{i} /\left(H_{i} \cap \sigma G\right)$ and, of course, $H_{i} /\left(H_{i} \cap \sigma G\right)$ $\cong H_{i} \sigma G / \sigma G$. This completes the proof.

\section{The proof of $\mathbf{1 . 6}$}

Throughout this section, $G$ is a finite monolithic group and $\mathscr{D}, \mathfrak{D}^{*}$ are defined as in 1.5.

Proof OF 1.61. Suppose that $\sigma^{*} G \leqq \sigma G$. If $\sigma^{*} G=1$ and $D \in D^{*}$, then $|D| \geqq\left|D / \sigma^{*} D\right|=|G| \sigma^{*} G|=| G \mid$. If $\sigma^{*} G=\sigma G$ and $D \in D^{*}$, then $\sigma G$, and hence also $\sigma D$, is abelian; so $\sigma^{*} D \geqq \sigma D,|D| \geqq|\sigma D|\left|D / \sigma^{*} D\right|=|\sigma G|\left|G / \sigma^{*} G\right|$ $=|G|$. In either case, we have a contradiction, for $D$ is a proper factor of $G$ : thus $\mathfrak{D}^{*}$ must be empty and so $G \notin \operatorname{var} \mathfrak{D}^{*}$. Now 1.51 shows that $G$ is critical.

Proof of 1.62. Let $\Phi(G)=1$; then $\sigma G \$ M$ for some maximal subgroup $M$ of $G$. If $\sigma G$ is non-abelian, then $\sigma^{*} G=1$ and so 1.61 gives that $G$ is critical. If $\sigma G$ is abelian, then $M \cap \sigma G$ is centralized by $\sigma G$ and, of course, normalized by $M$ : hence $M \cap \sigma G$ is normalized by $M \sigma G$. But $M \sigma G=G$, so $M \cap \sigma G \leqq G$, $M \cap \sigma G<\sigma G$ : it follows that $M \cap \sigma G=1$. Note that $\sigma^{*} G=\sigma G\left(M \cap \sigma^{*} G\right)$; $M \cap \sigma^{*} G$ is also centralized by $\sigma G$ and normalized by $M$, so that $M \cap \sigma^{*} G \leqq G$, and $\left(M \cap \sigma^{*} G\right) \cap \sigma G \leqq M \cap \sigma G=1$ : thus $M \cap \sigma^{*} G=1, \sigma^{*} G=\sigma G$. Now 1.61 gives that $G$ is critical.

In preparation for the proof of 1.63 we need a lemma:

(5.1) Let $X$ be a finite group, $Z$ a nilpotent normal subgroup of $X$, and $i$ a non-negative integer. Then $\left[\sigma_{i+1} X, Z\right] \leqq \sigma_{i} X$; in particular, $Z \leqq \sigma^{*} X$. 
Proof. Let $Y / \sigma_{i} X$ be a minimal normal subgroup of $X / \sigma_{i} X$. Then either $Y / \sigma_{i} X \cap Z \sigma_{i} X / \sigma_{i} X=1$ or, as $Z \sigma_{i} X / \sigma_{i} X$ is nilpotent, $1<$ $Y / \sigma_{i} X \cap \zeta_{1}\left(Z \sigma_{i} X / \sigma_{i} X\right) \leqq X / \sigma_{i} X$ and so $Y / \sigma_{i} X \leqq \zeta_{1}\left(Z \sigma_{i} X / \sigma_{i} X\right)$. In either case, $Y / \sigma_{i} X$ centralizes $Z \sigma_{i} X / \sigma_{i} X$. As this holds for every choice of $Y / \sigma_{i} X$, it follows that $\sigma\left(X / \sigma_{i} X\right)$ centralizes $Z \sigma_{i} X / \sigma_{i} X$; that is, $\left[\sigma_{i+1} X, Z\right] \leqq \sigma_{i} X$ as required.

Proof of 1.63. Let $\Phi(G)=\sigma^{*} G$ and suppose that $G$ is not critical. By 1.51, $G \in \operatorname{var} \mathfrak{D}^{*}$; let $H / K \in \mathfrak{D}^{*}, K \unlhd H<G$, and let $M$ be a maximal subgroup of $G$ containing $H$. Since $\Phi(G)$ is nilpotent, 5.1 implies that $(H \cap \Phi(G)) K / K \leqq \sigma^{*}(H / K)$ and so $H \cap \Phi(G) \leqq \sigma^{*}(H \div K)$. Since $H /(H \cap \Phi(G)) \cong \Phi(G) H / \Phi(G)$ and $\Phi(G) H \leqq M$, it follows that

$$
\begin{aligned}
\left|D / \sigma^{*} D\right| & \leq\left|H / \sigma^{*}(H \div K)\right| \leqq|H /(H \cap \Phi(G))| \\
& =|\Phi(G) H / \Phi(G)| \leqq|M / \Phi(G)|<|G / \Phi(G)|=\left|G / \sigma^{*} G\right|,
\end{aligned}
$$

so that $\sigma D$ is not similar to $\sigma G$, contrary to the definition of $\mathfrak{D}^{*}$.

For the proof of 1.64, we need one more lemma:

(5.2) Let $X$ be a finite group, $Y$ a subgroup and $Z$ a nilpotent normal subgroup of $X$ such that $X=Y Z$. Then, for every non-negative integer $i$, $\sigma_{i} X \cap Y \cap Z \leqq \sigma_{i} Y$.

Proof: by induction on $i$. The initial step is obvious. Define $W_{j}=\sigma_{j} X$ $\cap Y \cap Z$ for every non-negative integer $j$, and suppose that $W_{i} \leqq \sigma_{i} Y$. Consider $\sigma\left(X / \sigma_{i} X\right)$ as a group with $X$ as group of operators acting on it, each element $x$ of $X$ operating as the automorphism of $\sigma\left(X / \sigma_{i} X\right)$ induced by the inner automorphism of $X$ associated with $x$. Then $\sigma\left(X / \sigma_{i} X\right)$ is the product of its minimal $X$-admissible normal subgroups, and consequently (cf. $\S 61$ of Kurosh [8]) every $X$-admissible normal subgroup of $\sigma\left(X / \sigma_{i} X\right)$ is a product of minimal $X$-admissible normal subgroups of $\sigma\left(X / \sigma_{i} X\right)$. Since $X$ induces every inner automorphism of $\sigma\left(X / \sigma_{i} X\right)$, this means that if $A$ is an $X$-admissible subgroup of $\sigma\left(X / \sigma_{i} X\right)$, then $A$ is the product of the minimal $X$-admissible subgroups of $A$. Now $W_{i+1} \sigma_{i} X \leqq \sigma_{i+1} X$, so by $\mathbf{5 . 1}$

$$
\left[W_{i+1} \sigma_{i} X, Z\right] \leqq \sigma_{i} X,
$$

and thus $W_{i+1} \sigma_{i} X$ is normalized by $Z$; as $W_{i+1} \leqq Y$ and $\sigma_{i} X \leqq X$, it follows that $W_{i+1} \sigma_{i} X \leqq Y Z=X$. Put $A=W_{i+1} \sigma_{i} X / \sigma_{i} X$ : then $A$ is an $X$ admissible subgroup of $\sigma\left(X / \sigma_{i} X\right)$, so that this $A$ is the product of its minimal $X$-admissible subgroups. But (5.3) shows that $Z$ acts trivially on $A$, so that the $X$-admissible subgroups of $A$ are precisely the $Y$-admissible ones: $A$ is the product of its minimal $Y$-admissible subgroups. The isomorphism theorem gives that $A$ is $Y$-isomorphic to $W_{i+1} /\left(W_{i+1} \cap \sigma_{i} X\right)$, that is, to $W_{i+1} / W_{i}$ : thus $W_{i+1} / W_{i}$ is the product of its minimal $Y$-admissible sub- 
groups. In other words, $W_{i+1} / W_{i}$ is a product of minimal normal subgroups of $Y / W_{i}$, so that $W_{i+1} / W_{i} \leqq \sigma\left(Y / W_{i}\right)$. Applying 2.5 and $W_{i} \leqq \sigma_{i} Y$, and then using 2.1, we get now that

$$
W_{i+1} / W_{i} \leqq \sigma\left(Y / W_{i}\right) \leqq \sigma\left(Y / W_{i} \div \sigma_{i} Y / W_{i}\right)=\sigma\left(Y \div \sigma_{i} Y\right) / W_{i}=\sigma_{i+1} Y / W_{i}
$$

whence $W_{i+1} \leqq \sigma_{i+1} Y$. This completes the inductive step.

Proof of 1.64. Suppose that $\sigma^{*} G$ is nilpotent and has only one maximal $G$-normal subgroup $L$. In view of 1.61 , we assume that $\sigma^{*} G>\sigma G$. Then there exists a positive integer $n$ such that $\sigma^{*} G \leqq \sigma_{n} G$ but $\sigma^{*} G \leqq \sigma_{n+1} G$. Note that in this case $\sigma_{n} G \cap \sigma^{*} G=L$. We shall show that, for every $D$ in $D^{*}, \sigma^{*} D \leqq \sigma_{n} D$; by $2.9, \sigma_{n}$ is a subdirect function, and so this will prove that $G$ is critical: otherwise we would have a contradiction to 1.54. Let $D$ be an arbitrary element of $\mathfrak{D}^{*}, D=H / K, K \unlhd H<G$. As $\left(H \cap \sigma^{*} G\right) K / K$ is a nilpotent normal subgroup of $H / K, 5.1$ guarantees that $H \cap \sigma^{*} G \leqq \sigma^{*}(H \div K)$. Thus $\left|G / \sigma^{*} G\right|=\left|D / \sigma^{*} D\right|=\left|H / \sigma^{*}(H \div K)\right| \leqq\left|H /\left(H \cap \sigma^{*} G\right)\right|$ and so $\left|H \sigma^{*} G\right|=$ $\left|H /\left(H \cap \sigma^{*} G\right)\right|\left|\sigma^{*} G\right| \geqq|G|:$ it follows that $H \sigma^{*} G=G$ and $H \cap \sigma^{*} G=$ $\sigma^{*}(H \div K)$. Let $M$ be a maximal subgroup of $G$ containing $H$. Then $M \sigma^{*} G=G$, so $\sigma^{*} G \$ M, M \cap \sigma^{*} G<\sigma^{*} G$. Since $\sigma^{*} G$ is nilpotent, the normalizer $N$ of $M \cap \sigma^{*} G$ in $\sigma^{*} G$ is larger than $M \cap \sigma^{*} G$. As $M$ normalizes $M \cap \sigma^{*} G$, it normalizes $N$ : so $M N$ is a subgroup; but $N \$ M$, so $M N=G$. Thus $M \cap \sigma^{*} G \leqq G$, and therefore we must have $M \cap \sigma^{*} G \leqq L$. By what has been proved so far, $\sigma^{*}(H \div K)=H \cap \sigma^{*} G \leqq M \cap \sigma^{*} G \leqq L \leqq \sigma_{n} G$, so $\sigma^{*}(H \div K)$ $=\sigma_{n} G \cap H \cap \sigma^{*} G$ : here 5.2 can be applied to get that $\sigma^{*}(H \div K) \leqq \sigma_{n} H$, and from this 2.5 yields $\sigma^{*}(H \div K) \leqq \sigma_{n}(H \div K)$. This is the same as $\sigma^{*} D \leqq \sigma_{n} D$, and so the proof is complete.

Proof of 1.65 . In view of 1.61 , assume that $\sigma^{*} G>\sigma G$. Since $G$ is monolithic and $\sigma^{*} G$ is abelian, $\sigma^{*} G$ must be a $p$-group for some prime $p$; as $\sigma^{*} G$ has no element of order $p$ outside $\sigma G$, it follows that $\sigma\left(\sigma^{*} G\right)=\sigma G$. Let $p^{k+1}$ denote the exponent of $\sigma^{*} G$; we have that $k>0$. The set $\left\{g^{p^{*}}: g \in \sigma^{*} G\right\}$ is a non-trivial characteristic subgroup of $\sigma^{*} G$ contained in $\sigma\left(\sigma^{*}\right) G$ : hence $\sigma G=\sigma\left(\sigma^{*} G\right)=\left\{g^{p^{k}}: g \in \sigma^{*} G\right\}$. Thus every element of $\sigma\left(\sigma^{*} G\right)$ is a $p^{k}$ th power in $\sigma^{*} G$, and so $\sigma^{*} G$ must be a direct product of cyclic groups of order $p^{k+1}$. Hence $g \Phi\left(\sigma^{*} G\right) \rightarrow g^{p^{k}}$ is a $G$-isomorphism of $\sigma^{*} G / \Phi\left(\sigma^{*} G\right)$ onto $\sigma G$, and consequently $\Phi\left(\sigma^{*} G\right)$ is a maximal $G$-normal subgroup of $\sigma^{*} G$. On the other hand, if $M$ is any maximal $G$-normal subgroup of $\sigma^{*} G$, then $\sigma^{*} G / M$ is elementary abelian and therefore $M \geqq \Phi\left(\sigma^{*} G\right)$. This proves that $\Phi\left(\sigma^{*} G\right)$ is the only maximal $G$-normal subgroup of $\sigma^{*} G$ : now 1.64 gives that $G$ is critical.

Proof of 1.66. In view of 1.61 , assume $\sigma^{*} G>\sigma G$ : then $\sigma G$ is contained in the Fitting subgroup $F$ of $G$ and, as $G$ is monolithic, $F$ is a $p$-group for 
some prime $p$. By the assumption of $1.66, F$ is abelian. Now $\sigma F$ is an elementary abelian normal subgroup of $G$ (contained in and therefore) centralized by every (abelian) Sylow $p$-subgroup of $G$ : as $G$ is monolithic, Maschke's Theorem implies that $\sigma F=\sigma G$. In view of 1.65 , it suffices to prove that $F=\sigma^{*} G$. For a proof by contradiction, suppose that $F<\sigma^{*} G$ : then $\sigma^{*} G$ is not a $p$-group. Let $H$ be minimal among those normal subgroups of $G$ which are contained in $\sigma^{*} G$ and are not $p$-groups. If $g$ is any non-trivial element of $\sigma G$, then $g$ is of order $p$ and is in the centre of $H$ : since the Sylow $p$-subgroups of $H$ are abelian, it follows that the image of $g$ under the transfer of $H$ into any Sylow $p$-subgroup of $H$ is non-trivial (cf. Lemma 14.4.1 in M. Hall [2]). Consequently, $H$ has proper normal subgroups of $p$-power index; the intersection $K$ of all these is normal in $G$ and still has $p$-power index in $H$ : thus $K$ is not a $p$-group, contrary to the minimal choice of $H$. This completes the proof.

\section{The proof of 1.8}

Let $G$ be a critical group, $\sigma^{*} G$ abelian, $\sigma G$ a $p$-group, and suppose that $G / \sigma^{*} G$ has a $p$-soluble minimal normal subgroup $M / \sigma^{*} G$. Since $G$ is monolithic, $\sigma^{*} G$ is a $p$-group. On account of 5.1, $M$ cannot be a $p$-group: hence $M / \sigma^{*} G$ is a $p^{\prime}$-group. Thus, by the Schur-Zassenhaus Theorem (see e.g. M. Hall [2], p. 224) $\sigma^{*} G$ must have a complement $X$ in $M$. Let $N$ be minimal among those normal subgroups of $G$ which are contained in $\sigma^{*} G$ and for which $N X$ is normal in $G$. By Theorem 1 of Higman [3], $N$ has a complement $L$ in $G$. Now $L \cap \sigma^{*} G$ is normalized by $L$ and centralized by $N$ (as $N$ is abelian): so $L \cap \sigma^{*} G \leqq L N=G$. On the other hand, $\left(L \cap \sigma^{*} G\right) \cap \sigma G \leqq$ $L \cap N=1$, for $\sigma G \leqq N$ would imply $N=1,1<X \triangleleft G, X \cap \sigma G \leqq$ $X \cap \sigma^{*} G=1$ a contradiction. Thus $L \cap \sigma^{*} G$ is a normal subgroup of $G$ which avoids the socle of $G$ : we must have $L \cap \sigma^{*} G=1$. Obviously, $G=L N \leqq$ $L \sigma^{*} G$. Now if $\dot{N}_{1}, N_{2}$ were two distinct maximal $G$-normal subgroups of $\sigma^{*} G$, we would have that $L N_{1} N_{2}=G\left(\right.$ as $\left.N_{1} N_{2}=\sigma^{*} G\right), L N_{1}<G, L N_{2}<G$, and $\left[N_{1}, N_{2}\right]=1$, and so by Theorem 9 in Higman [5] (cf. also Theorem 4 of Powell [10]) $G$ could not be critical.

\section{References}

[1] Reinhold Baer, Endlich definierbare gruppentheoretische Funktionen. Math. Z. 87 (1965), $163-213$.

[2] Marshall Hall, Jr, The theory of groups. Macmillan, New York, 1959.

[3] Graham Higman, Complementation of abelian normal subgroups. Publ. Math. Debrecen 4 (1956), 455-458.

[4] Graham Higman, Some remarks on varieties of groups. Quart. J. Math. Oxford Ser. (2) 10 (1959), 165-178. 
[5] Graham Higman, Identical relations in finite groups. Conv. Internaz. di Teoria dei Gruppi Finiti (Firenze, 1960), pp. 93-100. Edizione Cremonese, Rome, 1960.

[6] L. G. Kovács and M. F. Newman, Minimal verbal subgroups. To appear in Proc. Cambridge Philos. Soc. 62 (1966).

[7] L. G. Kovács and M. F. Newman, Cross varieties of groups. To appear in Proc. Roy. Soc. Ser. A 1966.

[8] A. G. Kurosh, The theory of groups, vol. II. Chelsea, New York, 1956.

[9] Sheila Oates and M. B. Powell, Identical relations in finite groups. J. Algebra 1 (1964), $11-39$.

[10] M. B. Powell, Identical relations in finite soluble groups. Quart. J. Math. Oxford Ser. (2) 15 (1964), 131-148.

[11] P. M. Weichsel, A decomposition theory for finite groups with applications to p-groups. Trans. Amer. Math. Soc. 102 (1962), 218-226.

[12] P. M. Weichsel, On critical p-groups. Proc. London Math. Soc. (3) 14 (1964), 83-100.

\section{Australian National University Canberra}

